\title{
Entropy of $\mathrm{H}_{2} \mathrm{O}$ Wetting Layers ${ }^{\dagger}$
}

\author{
Peter J. Feibelman*, and Ali Alavi ${ }^{\S}$ \\ Sandia National Laboratories, Albuquerque, New Mexico 87185-1413 and Department of Chemistry, \\ University of Cambridge, Lensfield Road, Cambridge CB2 1EW, United Kingdom
}

Received: January 6, 2004; In Final Form: April 26, 2004

\begin{abstract}
Proton order is less constrained in a fully $\mathrm{H}$-bonded, adsorbed $\mathrm{H}_{2} \mathrm{O}$ monolayer than in bulk ice. Thus, though weakly, configurational entropy favors wetting by deposited $\mathrm{H}_{2} \mathrm{O}$ over formation of 3-D crystalline mounds. A Pauling-type estimate yields a $0 \mathrm{~K}$ adlayer entropy of $\sim 1 /{ }_{2} k_{\mathrm{B}} \ln (9 / 2)$, almost twice that of ice Ih. Thus, at $150 \mathrm{~K}$, where periodic adlayers on metals are observed, residual entropy reduces their free energies relative to a 3-D ice crystal by $4.5 \mathrm{meV} / \mathrm{molecule}$, or $\sim 3 \%$ of the cost of the adlayers' broken H-bonds. A Debye model implies that vibrational entropy contributes no more than another $4.4 \mathrm{meV} / \mathrm{molecule}$ to the free energy preference for wetting, at $150 \mathrm{~K}$. This information, though only a bound because of substantial uncertainty in measured adlayer Debye temperatures, is nonetheless sufficient to conclude that $T=0 \mathrm{~K}$ energies dominate the free energy balance between wetting and mound formation.
\end{abstract}

\section{Introduction}

The need to count the low-energy configurations of a protondisordered, hydrogen-bonded crystal like ice Ih to evaluate its configurational entropy has spawned the development of "ice models" and studies of their subtle correlations. Though typically two-dimensional, such models are neither designed to represent, nor used to understand, real 2-D systems. Thus, 2-D "square ice," a model solved accurately by Nagle ${ }^{1}$ and exactly by Lieb, ${ }^{2}$ is not intended to represent a wetting layer, but expressly, and by construction, a three-dimensional bulk ice crystal, with four bond directions and six possible arrangements of two $\mathrm{H}$ atoms per $\mathrm{O}$.

In contrast, a fully H-bonded, 2-D wetting layer has just three bonds per two $\mathrm{O}$ atoms plus one "free" $\mathrm{H}$ (or D), as seen in Figures 1 and 2. The residual entropy of a real, two-dimensional layer of water molecules is therefore different from that of the usual ice models, and indeed is almost twice as large! This is a big effect compared to that of the subtle correlations found for square ice, ${ }^{1,2}$ which change its entropy by less than $0.5 \%$.

There is ample reason to study real, 2-D, water molecule layers. A host of important phenomena, e.g., flow through nanopores, corrosion, and condensation, are influenced by the wetting of solid surfaces. Learning to predict what surfaces will wet, how near-surface water molecules will be arranged, and how surface chemistry will affect dynamic phenomena in adjacent water or ice would be major steps forward.

Density functional theory ${ }^{3}$ (DFT) total energy calculations have obvious potential for providing such understanding. However, it is not clear that approximations inherent in current DFT implementations are accurate enough to support firm conclusions. ${ }^{4}$ For a weakly interacting, closed-shell molecule like $\mathrm{H}_{2} \mathrm{O}$, one concern is that DFT in its current form treats van der Waals forces crudely (at best). ${ }^{5}$ Another is that the quantum nature of the proton might be nonnegligible. ${ }^{6}$ Our focus here is that DFT total energies are ordinarily not free energies. Thus, strictly speaking, the results of DFT calculations describe

† Part of the special issue "Gerhard Ertl Festschrift".

$\div$ Sandia National Laboratories.

$\S$ University of Cambridge.

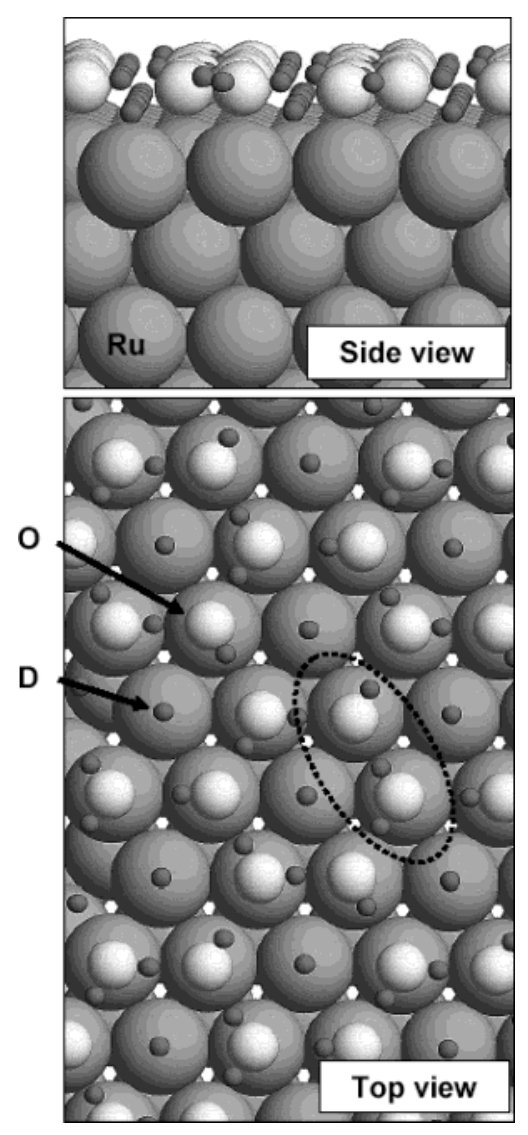

Figure 1. Side and top views of a half-dissociated $\mathrm{D}_{2} \mathrm{O}$ layer on $\mathrm{Ru}(0001)$. The dotted ellipse makes plain that for every two $\mathrm{O}$ atoms there are three H-bonds.

thermodynamic stability only at $0 \mathrm{~K}$, and entropy estimates, at a minimum, are required to extend to laboratory temperatures.

At the lowest temperatures, the dominant contribution to the entropy of an ice crystal is the residual, configurational term associated with proton disorder in the H-bonded network. ${ }^{7}$ In 1935, Pauling proposed an estimate of this term for bulk ice Ih, $k_{\mathrm{B}} \ln (3 / 2)$, which proved remarkably accurate. ${ }^{8}$ Here, we begin by generalizing his simple approach to ice "slabs" 
free $D$ atoms up
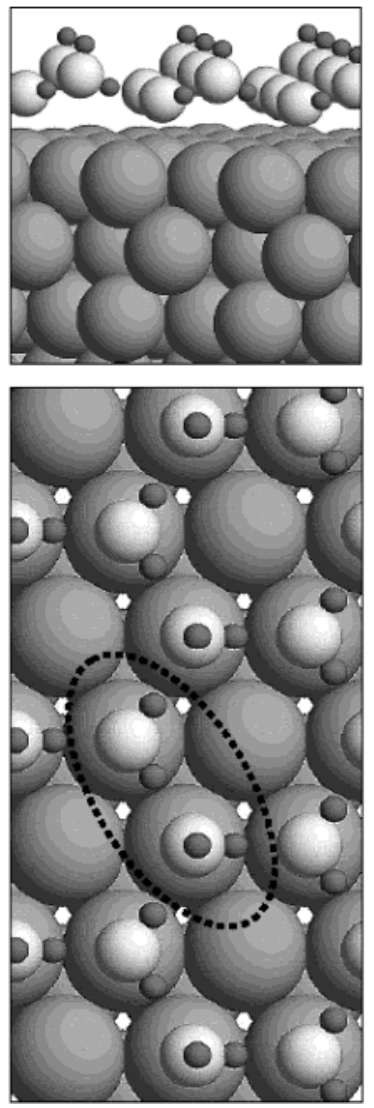
Figure 2. Side and top views of $\mathrm{D}$-up and $\mathrm{D}$-down $\mathrm{D}_{2} \mathrm{O}$ adlayers on
$\mathrm{Ru}(0001)$. The dotted ellipses show, as in Figure 1 , that for every two $\mathrm{O}$ atoms there are three $\mathrm{H}$-bonds.

comprised of $L$ layers, thereby learning that the configurational entropy associated with forming an ice surface is positive but small relative to the H-bond energy divided by $273 \mathrm{~K}$.

If one could neglect vibrational entropy, this would mean that a two-dimensional structure found via DFT calculations to be tenths of an electronvolt shy of stably wetting a surface at 0 $\mathrm{K}^{9}$ would be nonwetting at any temperature. However, at the maximum temperatures where periodic water adlayers are observed, in the neighborhood of $150 \mathrm{~K}$, the vibrational entropy of ice Ih is roughly 6 times larger than its residual entropy. ${ }^{10}$ Thus, to analyze the reliability of DFT in predicting wetting, in this temperature regime, an estimate of adlayer vibrational entropy is required.

The origin of this project is lingering doubt on how to interpret the only quantitative experimental structure of a water layer on a solid, Held and Menzel's low energy electron diffraction (LEED) result for $\sqrt{3} \times \sqrt{3}-R 30^{\circ}-$ $\mathrm{D}_{2} \mathrm{O} / \mathrm{Ru}(0001) .{ }^{11}$ This structure has long seemed puzzling. Confounding the sense that the $\mathrm{D}_{2} \mathrm{O}$ adlayer should adopt a "puckered-hexagonal" arrangement, with $\mathrm{O}$ atoms residing in two planes separated by $\sim 1 \AA$, as in a single basal layer of ice Ih, ${ }^{12}$ Held and Menzel determined that the $\mathrm{O}$ atoms of $\sqrt{3} \times$ $\sqrt{3}-R 30^{\circ}-\mathrm{D}_{2} \mathrm{O} / \mathrm{Ru}(0001)$ are close to coplanar.

Heavy water on $\mathrm{Ru}(0001)$ thus offers a unique opportunity not only to test whether DFT structural parameters agree with experiment, but also to explore the physics that causes the adlayer to violate the expectation that it be "ice-like." An effort of this sort by one of us, involving both first-principles calculations of lattice energy ${ }^{9}$ and an estimate of zero-point energy, ${ }^{13}$ pointed to the likelihood that Held and Menzel's periodic $\mathrm{D}_{2} \mathrm{O}$ adlayer was constituted of a hydrogen-bonded arrangement of $\mathrm{D}_{2} \mathrm{O}$ molecules and OD fragments, together with $\mathrm{D}$ atoms bonded independently to $\mathrm{Ru}$ atoms. Adsorption energies for adlayers made purely of intact $\mathrm{D}_{2} \mathrm{O}$ molecules were found to be $\sim 0.2 \mathrm{eV}$ smaller and had the incorrect, puckered structure. ${ }^{9}$

In the present work, we question whether these $0 \mathrm{~K}$ theoretical results are meaningful relative to experiments carried out at 150 $K$. In principle, if the entropy of bulk ice were larger than that of a two-dimensional adsorption structure, the answer would be "certainly yes." At finite temperature, the free energy of the bulk crystal would drop faster than that of the adlayer. On the other hand, if the two-dimensional film had the larger entropy, the ordering of crystal and adlayer free energies could conceivably reverse.

To learn which situation obtains, we begin by applying Pauling's method, in the following section, to estimate an adlayer's configurational entropy. The result is that, although it exceeds that of bulk ice Ih, the excess is too small to affect wetting at any temperature of interest (i.e., such that the water has not evaporated). In section III, an estimate of an adlayer's vibrational entropy is shown to leave this initial conclusion unaltered. These results are recapitulated and discussed in section IV. Last, since the excess configurational entropy of two-dimensional water adlayers compared to bulk ice may be viewed as surprising, we provide computational support, in three appendices, for the Pauling-type counting that leads to this result. We provide explicit algebraic details and low-temperature asymptotic results for the vibrational entropy in the Debye model, in a fourth appendix.

\section{Residual Entropy of H-Bonded Arrangements of Water Molecules}

Pauling's estimate of the ice Ih residual entropy is based on the following assumptions (the first two of which are known as the "ice rules" 8,14 ):

1. Two $\mathrm{H}$ atoms belong to each $\mathrm{O}$ atom.

2. Precisely one $\mathrm{H}$ atom lies on the line connecting any two $\mathrm{O}$ atoms.

3. The two H's belonging to each $\mathrm{O}$ atom can occupy its four tetrahedral bond directions in $4 ! /(2 !)^{2}$, or 6 , ways.

4. All ice crystals obeying the ice rules have the same, low energy.

5. If one randomly assigns $\mathrm{H}$ atoms to $\mathrm{O}-\mathrm{O}$ bonds, the probability that no bond will contain zero or two $\mathrm{H}$ atoms, but just one, equals $1 / 2$, per bond.

According to these assumptions, the number of ways of forming equal energy bulk ice crystals with $N \mathrm{O}$ atoms and $2 \mathrm{~N}$ bonds is $6^{N}(1 / 2)^{2 N}$, or $(3 / 2)^{N}$. This means the (per water molecule) configurational entropy of ice, given by the logarithm of this number times the Boltzmann constant, is $S / N=k_{\mathrm{B}} \ln$ $(3 / 2)$.

The same result obtains for square ice, which, like ice Ih, has two $\mathrm{H}$ 's and four bond directions per $\mathrm{O}$ atom and one $\mathrm{H}$ on every $\mathrm{O}-\mathrm{O}$ bond. For a single ice adlayer, as seen in Figures 1 and 2 , the entropy is larger because $1 / 4$ of the $\mathrm{H}$ atoms do not participate in $\mathrm{H}$-bonding. Whether the non- $\mathrm{H}$-bonding $\mathrm{H}$ atoms are dissociated and attached directly to the metal (Figure 1), or are part of intact water molecules and point into the vacuum or toward the metal (Figure 2), the constraint that there be one $\mathrm{H}$ on each $\mathrm{O}-\mathrm{O}$ bond has less effect.

Specifically, with just three $\mathrm{H}$-bonds for every two $\mathrm{O}$ atoms in these cases, the number of ways one can make an adlayer 
with $N(\equiv 2 M)$ oxygen atoms is now $6^{2 M}(1 / 2)^{3 M}$ or $(36 / 8)^{N / 2}$. This application of Pauling's logic implies that the adlayer entropy is $1 / 2 k_{\mathrm{B}} \ln (9 / 2)$, exceeding that of bulk ice by $1 / 2 k_{\mathrm{B}}$ $\ln (2)=0.346 k_{\mathrm{B}}$, on a per molecule basis, or, alternately, by a factor of 1.854 . The reason is simply that the adlayer is freer to arrange itself than the bulk crystal by virtue of having fewer $\mathrm{H}$-bonds per $\mathrm{O}$ atom.

One can apply similar logic to a hypothetical, wetting multilayer, leading to a formula that conveniently connects the results for a monolayer and for bulk ice. Specifically, in an ice slab comprised of $L$ layers, with $2 M$ oxygen atoms per layer, there are $M(4 L-1)$ hydrogen bonds. ${ }^{15}$ The number of ways one can construct an $L$-layer slab is therefore $6^{2 M L}(1 / 2)^{M(4 L-1)}$ $=(3 / 2)^{2 M L} 2^{M}$, and the residual entropy per molecule is $k_{\mathrm{B}}[\ln$ $(3 / 2)+\ln (2) / 2 L]$. This approaches Pauling's bulk ice result for large $L$ and rises to the monolayer value when $L=1 .{ }^{16}$

From a quantitative perspective, though the residual entropy of an adlayer is greater than that of ice, and therefore favors wetting as the temperature rises from $0 \mathrm{~K}$, the effect is small. At $T=150 \mathrm{~K}, k_{\mathrm{B}} T=12.9 \mathrm{meV}$. Thus the configurational entropy contribution to an adsorbed monolayer's wetting free energy is only $4.5 \mathrm{meV}$, compared to a binding energy more than 50 times larger gained by forming a hydrogen bond. Residual entropy, accordingly, plays only a small role in deciding whether wetting will or will not occur, in general.

\section{Vibrational Entropy Contribution to Wetting}

The vibrational entropy of ice Ih increases with $T$, to roughly 6 times the residual component at $150 \mathrm{~K} .{ }^{10}$ Thus, an analysis of the reliability of DFT results in predicting wetting at laboratory temperatures inevitably requires an estimate of adlayer vibrational entropy. In what follows, we address this need in the simplest possible way, by applying a Debye model. This approach is justified by the dominant contribution of the long-wavelength, low-energy, acoustic modes of a two- or threedimensional system to its low-temperature vibrational entropy.

The only input needed is the Debye temperature, $\Theta_{\mathrm{D}}=\hbar \omega_{\mathrm{D}} /$ $k_{\mathrm{B}}$, which physically represents the highest frequency in the acoustic mode spectrum, and mathematically provides the cutoff for integrals over the modes. ${ }^{17}$ In practice, Debye temperatures are inferred from experiment, e.g., from low-temperature specific heat data, or from Debye-Waller factors. In general, they can be subject to significant error. For bulk ice Ih, the experimental consensus is a value in the range $\Theta_{\mathrm{D}}=218-225 \mathrm{~K} \cdot{ }^{18,19}$ For the adlayer Debye temperature, there is considerably more uncertainty. ${ }^{20,21}$

We begin by showing evidence that the Debye approach accounts for the vibrational entropy of bulk ice Ih to better than $50 \%$. Based on this result, it is reasonable to assume that a twodimensional Debye model will describe the vibrational entropy of an adlayer at a comparable level of accuracy. At the end of this section we thereby arrive at a rough estimate of the 2-D vs 3-D difference-which turns out to be comparable in magnitude (or smaller, depending on the experimental value used for the adlayer's $\Theta_{\mathrm{D}}$ ) to the difference of the residual entropies at $150 \mathrm{~K}$.

For a $d$-dimensional system (see Appendix D), in the harmonic approximation, the Debye model expression for the vibrational entropy can be reduced to

$$
S(T) / N k_{\mathrm{B}}=d \int_{\Theta_{\mathrm{D}} / T}^{\infty} \frac{\mathrm{d} x}{\mathrm{e}^{x}-1}+d(d+1)\left(T / \Theta_{\mathrm{D}}\right)^{d} \int_{0}^{\Theta_{\mathrm{D}} / T} \frac{x^{d} \mathrm{~d} x}{\mathrm{e}^{x}-1}
$$

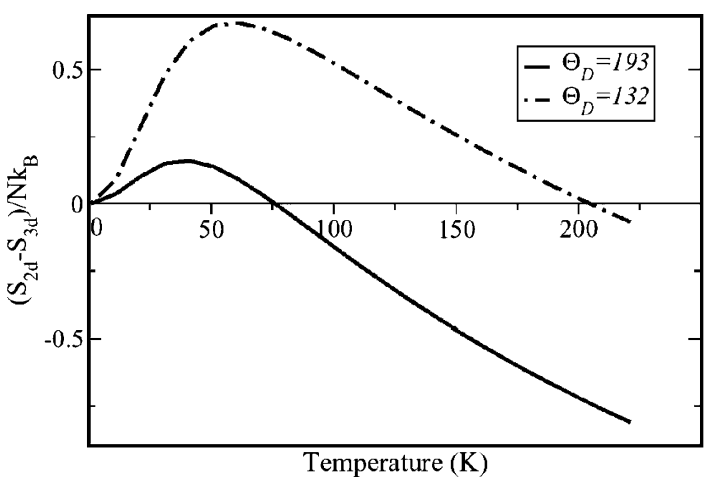

Figure 3. Computed vibrational entropy difference, in units of $k_{\mathrm{B}}$, versus temperature $(\mathrm{K})$, assuming a bulk ice Debye temperature of 218 $\mathrm{K}^{18}$ and an adlayer Debye temperature of either 132 or $193 \mathrm{~K}$.

For bulk ice $(d=3)$, assuming ${ }^{18} \Theta_{\mathrm{D}}=218 \mathrm{~K}$, we obtain vibrational entropies of 0.21 and $25.2 \mathrm{~J} \mathrm{~K}^{-1} \mathrm{~mol}^{-1}$ at 15 and $150 \mathrm{~K}$. Comparison to measured entropies of $0.31^{7}$ and $\sim 20^{10}$ at these temperatures shows that the Debye model gives tolerable agreement (though with plenty of room for improvement).

Accordingly, we proceed to evaluate the adlayer vibrational entropy in the Debye model, with confidence that the answer will be within $50 \%$ or so of the correct answer. Unfortunately, in this case, we must face the problem that the Debye temperature is poorly known, with values of 132 and $175 \mathrm{~K}$ given by the same group in two different experiments on thick ice layers, ${ }^{20}$ and $193 \pm 50 \mathrm{~K}$ estimated from a Debye-Waller factor observation on a single $\mathrm{D}_{2} \mathrm{O}$ layer on $\mathrm{Ru}(0001){ }^{21}$

This information is not entirely without content. There is a consensus that the adlayer Debye temperature is below that of bulk ice Ih, perhaps because there are fewer bonds per molecule in the former case, and on this basis, one expects the adlayer to have the higher vibrational entropy at sufficiently low $T$. On the other hand, the proportionality of the prefactors in eq 1 to $d$ favors the 3-D system, and thus one expects a crossover at some value of $T$.

To be more quantitative, we bracket the Debye model difference between bulk ice and adlayer vibrational entropies in Figure 3 by examining results obtained over the range of suggested values of the adlayer $\Theta_{D}$. The results in the figure show that the crossover temperature above which the vibrational entropy of bulk ice exceeds the adlayer's is a decreasing function of the adlayer $\Theta_{\mathrm{D}}$. Thus if we use $\Theta_{\mathrm{D}}{ }^{\mathrm{b}}=218 \mathrm{~K}$ for bulk ice, ${ }^{18}$ in eq 1 , and accept Held's rough value for the water adlayer, $\Theta_{\mathrm{D}}{ }^{\mathrm{a}}=193 \mathrm{~K},{ }^{21}$ then at $150 \mathrm{~K}$ the vibrational entropy of bulk ice exceeds that of $\mathrm{D}_{2} \mathrm{O} / \mathrm{Ru}(0001)$ by $0.47 k_{\mathrm{B}}$, overcompensating the adlayer's excess configurational entropy by $0.12 k_{\mathrm{B}}$. If, on the other hand, we assume that $\Theta_{D}{ }^{a}=132 \mathrm{~K}$ is a more realistic adlayer Debye temperature ${ }^{20}$ then the adlayer vibrational entropy exceeds that of bulk ice up to $205 \mathrm{~K}$, and at $150 \mathrm{~K}$ adds $0.26 k_{\mathrm{B}}$ to the adlayer's excess configurational entropy.

\section{Discussion}

From a practical perspective, what is important about these results is not the obvious fact that a tighter experimental bound on the adlayer Debye temperature is needed to pin down even the sign of its excess entropy relative to ice Ih at laboratory temperatures. Rather, it is that, for any reasonable choice of $\Theta_{\mathrm{D}}{ }^{\mathrm{a}}$, the contribution of $\Delta S$ to the free energy balance that determines whether wetting is favorable is small on the scale of tenths of an electronvolt, at laboratory temperatures. Thus, the restriction of ordinary DFT calculations to $T=0 \mathrm{~K}$ should not hinder their ability to predict whether or how wetting occurs. 
A caveat, in this regard, is our tacit assumption, in computing the configurational entropy of a wetting layer, that its presumed perfect, H-bonded, $T=0 \mathrm{~K}$ structure does not change appreciably as the temperature of the system increases. Such structural changes would change the wetting layer's configurational entropy. On the other hand, it is hard to imagine that such changes would be large enough to alter the conclusion that the $T=0 \mathrm{~K}$ energies dominate the free energy balance between wetting and mound formation.

Ertlis encomium. Over his long and distinguished career, Professor Gerhard Ertl has led the effort to relate macroscale chemistry to atomic-scale processes. He has also stood as a role model for physical chemists, young and old. No "butterfly", flitting from scientific flower to flower, not interested in spending a career applying a single technique to whatever problem, Ertl's oeuvre offers an outstanding lesson on the value and importance of choosing important, representative problems, mustering the resources to attack them from many angles, and, over time, solving them definitively. We undertook the present work in this spirit. By addressing water-solid interactions from every angle, we hope ultimately to understand the statics and dynamics of this difficult and vastly important problem.

Acknowledgment. We are grateful to N. C. Bartelt for providing his transfer-matrix computer code. P.J.F. wishes to thank the Department of Chemistry of the University of Cambridge, and particularly Sir David King and his research group, for helping to make his visit to Cambridge stimulating and productive. This work was supported in part by the DOE Office of Basic Energy Sciences, Division of Material Sciences and Engineering. Sandia is a multiprogram laboratory operated by Sandia Corporation, a Lockheed-Martin Company, for the U.S. DOE under Contract No. DE-AC04-94AL85000.

\section{Appendices: Numerical Tests of the Pauling Approximation for Adlayers}

That there are more bonding configurations per molecule in a two-dimensional water adlayer than in bulk ice seems sufficiently surprising, at first blush, that computational checks on our logic are warranted. In the first three of the following appendices, accordingly, we report the results of several, ultimately reassuring, tests. In the last, we present details of the algebra leading to the Debye entropy expression of eq 1 and its asymptotic behavior.

A. A Pedagogical Issue. We begin with a pedagogical issueto understand the correlations that cause the residual entropy to depart from Pauling's estimate and, in particular, to grasp what makes his estimate a strong lower bound. ${ }^{22}$ To this end, neglecting chemical and geometric issues beyond the requirement of obeying the ice rules, consider how many ways one can make $N$-molecule linear chains, as against rings of water molecules.

The three-molecule case, illustrated in Figure 4, is both representative and simple. In the figure, heavy dots represent $\mathrm{O}$ atoms and arrows pointing away from some of them represent those of their $\mathrm{H}$ atoms that form donor $\mathrm{H}$-bonds to the neighboring O's. Some dots have no arrow emerging from them. They correspond to "double-acceptor" water molecules, neither of whose H's participates in H-bonding. There are also dots from which only a single arrow emerges. These correspond to single-donor, single-acceptor $\mathrm{H}_{2} \mathrm{O}$ 's. One of the $\mathrm{H}$ atoms of such molecules is free and can reside in either of two non-H-bonding tetrahedral orientations. Accordingly, for each single-arrow dot, the configuration shown actually represents two independent
Allowed cyclic chains and their degeneracies (adding to 28):

$\begin{array}{ccccc} & \text { A } & \text { B } & \text { C } & \text { degeneracy } \\ \text { 1) } & \leftarrow \bullet \rightarrow & \bullet \rightarrow & \bullet & 2 \\ 2) & \leftarrow \bullet & \bullet & \leftarrow \bullet & 2 \\ 3) & \leftarrow \bullet & \leftarrow \bullet & \bullet & 2 \\ 4) & \leftarrow \bullet & \leftarrow \bullet & \leftarrow \bullet & 8 \\ 5) & \bullet \rightarrow & \bullet \rightarrow & \bullet \rightarrow & 8 \\ 6) & \bullet \rightarrow & \bullet & \leftarrow \bullet & 2 \\ 7) & \bullet & \leftarrow \bullet & \bullet \rightarrow & 2 \\ 8) & \bullet & \leftarrow \bullet & \leftarrow \bullet \rightarrow & 2\end{array}$

Chains that obey the ice rules as linear but not as cyclic:

$\begin{array}{lcccc}9) & \leftarrow \bullet & \bullet & \bullet \rightarrow & 4 \\ 10) & \leftarrow \bullet & \bullet & \leftarrow \bullet & 1 \\ 11) & \leftarrow \bullet & \leftarrow \bullet & \bullet \rightarrow & 4 \\ 12) & \leftarrow \cdot & \leftarrow \bullet & \leftarrow \bullet & 4 \\ 13) & \bullet \rightarrow & \bullet & \bullet & 4 \\ 14) & \bullet \rightarrow & \bullet & \leftarrow \bullet & 4 \\ 15) & \bullet & \leftarrow \bullet & \bullet & 1 \\ 16) & \bullet & \leftarrow \bullet & \leftarrow \bullet & 4\end{array}$

Figure 4. Schematic of linear chains that obey the ice rules. Heavy dots represent $\mathrm{O}$ atoms. Arrows represent those of their $\mathrm{H}$ (or D) atoms which form donor H-bonds to neighboring O's. As explained in the text, "degeneracies" listed amount to 1 times a factor 2 for each water molecule that has only one $\mathrm{H}$ (or D) atom in the $\mathrm{H}$-bonding network. Cycle-forming chains are the ones in which there is either a rightpointing arrow on oxygen $\mathrm{C}$ and no left-pointing arrow on oxygen $\mathrm{A}$, or vice versa.

configurations and is therefore assigned a "degeneracy factor" of 2 .

With this explanation, it is straightforward to verify that there are 54 distinct chains of three water molecules, in all, just as Pauling-type reasoning predicts. ${ }^{23}$ Of these 54, as explicitly enumerated in Figure 4, 28 allow the chain to close on itself to form a cycle (i.e., a triangle). This is one more than the Paulingtype estimate, $6^{3} / 2^{3}=27 .{ }^{24}$

The reason there is one more is that, given the ice rules, fixing "A" to have a left-pointing $\mathrm{H}$ atom slightly increases the chance that " $\mathrm{C}$ " will not have a right-pointing $\mathrm{H}$ atom and, therefore, that a cycle can be formed. This "correlation" extends through the chain of three molecules and is the source of the violation of the Pauling probability argument. In 3-D ice Ih, according to Nagle, ${ }^{1}$ such correlations increase the residual entropy by roughly $0.5 \%$ relative to the Pauling estimate.

B. Estimating Residual Entropy via an "Embedded Cluster" Counting Method. One way to count the number of allowed ice bilayer configurations begins by viewing a bilayer as formed of two interpenetrating water-molecule sublattices, A and B, such that each site on the A sublattice is surrounded by three B-sublattice sites, and vice versa. Each site provides four possible locations for $\mathrm{H}$ atoms, arranged tetrahedrally, so that there are three equatorial $\mathrm{H}$ sites and one axial one.

Suppose our lattice has $2 N$ sites, $N$ of type A and $N$ of type B. We construct a configuration of $2 N$ molecules by first placing molecules on the A sites and then trying to place molecules on the B sites. Since the A sites are not connected, there are $6^{N}$ ways to place $\mathrm{H}$ atoms on them. Not all of these, however, allow $\mathrm{H}$ atoms to be associated with the $\mathrm{B}$ sites, because the ice rules come into play.

A key point in the counting is that on each B site, surrounded by a given configuration of A-type water molecules, there is a maximum of one way to form a water molecule. There might be no way, but there will not be more than one. This limit reduces the number of allowed configurations by a factor, which we can estimate by explicit calculations on finite clusters. 


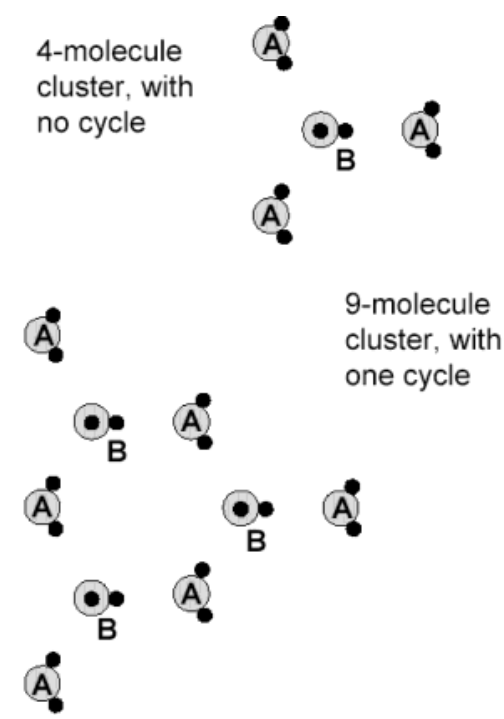

Figure 5. Schematic illustration of $2-\mathrm{D}$ water-molecule clusters containing four molecules and no cycle, and nine molecules with one cycle. Gray circles stand for $\mathrm{O}$ atoms and black dots for $\mathrm{H}$ atoms. The "A" and "B" labels correspond to the text in Appendix B.

TABLE 1: Computed Number of Configurations, Pauling Exponent, and Entropy Estimate, $S$, for Few-Molecule, Two-dimensional Water Clusters ${ }^{a}$

\begin{tabular}{lccc}
\hline \multicolumn{1}{c}{ cluster size } & $\begin{array}{c}\text { no. of valid } \\
\text { configurations }\end{array}$ & $\begin{array}{c}\text { Pauling } \\
\text { exponent }\end{array}$ & $S / k_{\mathrm{B}}$ \\
\hline $4=3^{(\mathrm{A})}+1^{(\mathrm{B})}$ & 162 & 2.121320 & 0.7520385 \\
$9=6^{(\mathrm{A})}+3^{(\mathrm{B})}$ & 19710 & 2.121805 & 0.7522671 \\
$16=10^{(\mathrm{A})}+6^{(\mathrm{B})}$ & 10806534 & 2.122055 & 0.7523850
\end{tabular}

${ }^{a}$ Convergence is rapid, with the entropy result for the 16-molecule cluster $0.05 \%$ greater than the Pauling-type estimate, 0.752039.

The idea is to embed a certain number $N_{\mathrm{B}}$ of $\mathrm{B}$ sites in a finite cluster of $N_{\mathrm{A}}$ A sites. Suppose we run over all $6^{N_{\mathrm{A}}}$ possible A-site configurations and count the number of valid B-site configurations; call this number $\zeta$. Then, $\zeta / 6^{N_{\mathrm{A}}}$ is the fraction of configurations consistent with the ice rules. The reduction in probability per $B$ site is therefore $\left(\xi / 6^{N_{\mathrm{A}}}\right)^{1 / N_{\mathrm{B}}}$, and the residual entropy per molecule can be estimated as

$$
S /\left(N k_{\mathrm{B}}\right) \approx \frac{1}{2} \ln \left[6\left(\xi / 6^{N_{\mathrm{A}}}\right)^{1 / N_{\mathrm{B}}}\right]
$$

The simplest example is a four-molecule cluster, with three A-site water molecules surrounding one B site (see Figure 5). There are $6^{3}=216$ a priori ways of configuring the three A-type molecules. However, of those, only $\zeta=162$ allow a water molecule to exist on the central B site, as can be checked explicitly by machine counting. As a result, the entropy per water molecule is $1 / 2 \ln \left(6 \times 162 / 6^{3}\right)=1 / 2 \ln (36 / 8)$, precisely in agreement with the Pauling-type estimate, as might be expected since the four-molecule cluster contains no cycles.

Matters are different, also as expected, for clusters large enough to contain "entropy-enhancing" rings of water molecules. We have studied two such clusters, with 9 and 16 molecules, and one and three complete rings, with results summarized in Table 1.

The nine-molecule cluster consists of three B sites, embedded among six A-type sites (see Figure 5). In this case, a computer count reveals that only 19710 of the $6^{6}=46656$ possible configurations of A-site molecules allow the placement of water molecules on the three B sites. Thus, the entropy estimate per molecule is $1 / 2 \ln \left(19710^{1 / 3} / 6\right)=\ln (2.121805 \ldots)$, slightly larger

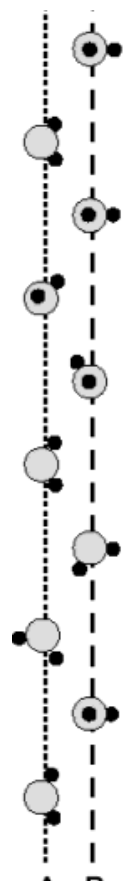

A B

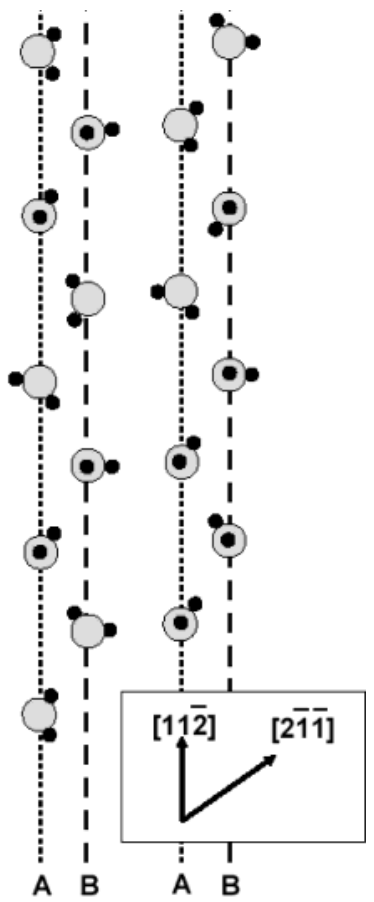

Figure 6. For transfer-matrix calculations, the adlayer is divided into A and B sublattices along the [112] direction and the lattice is built up by transferring along the [21] direction. The schematic shows a stripe width, or repeat distance of 4, along the [112] direction. Gray circles stand for $\mathrm{O}$ atoms and black dots for $\mathrm{H}$ atoms.

than the result for the four-molecule cluster. This increase in entropy reflects just the sort of correlations that were illustrated above for the three-molecule cycle.

For the 16-molecule cluster, 10806534 of the $6^{10}$ possible configurations of water molecules on $10 \mathrm{~A}$ sites allow the satisfaction of the ice rules when $\mathrm{H}_{2} \mathrm{O}$ 's are added to the $\mathrm{B}$ sites. Thus the 16-site cluster yields an entropy estimate of $k_{\mathrm{B}}$ $\ln (2.122055)$ per molecule. Evidently, the effect of correlations on the argument of the logarithm (the "Pauling exponent") is less than $0.1 \%$, which is negligible for most practical purposes. The Pauling-like estimate, $3 / \sqrt{2}=2.12132 \ldots$ is accurate enough.

C. Estimating Residual Entropy via the Transfer-Matrix Approach. As a last check, we used a transfer-matrix computer code kindly provided by N. C. Bartelt ${ }^{25}$ to estimate the residual entropy of an infinitely extended water adlayer. In this code one divides the extended water bilayer into one-dimensional "A" and "B" sublattices along the [112] direction, as in Figure 6. As in the cluster method, the water molecules of the A sublattice are, at the outset, free to adopt any orientation. However, only a fraction of these orientations allow the satisfaction of the ice rules, as one attempts to populate the B sublattice.

Bartelt's code begins by enumerating the $L_{N}$ inequivalent ways of populating a chain of sites along the [11ㄹ] direction, under the constraint that the arrangement be periodic with a repeat distance of $N$ sites. (Inequivalent arrangements are those that are not identical under a shift of the origin of the repeating cell.) For each of the inequivalent structures, $i$, the code then runs through the list once again to see which of the $L_{N}$ arrangements, $j$, is suitable for the next A-sublattice chain. Suitability is determined by attempting to fill the intervening B-sublattice chain while obeying the ice rules. If the ice rules permit a filling of the B-sublattice chain, then the transfer-matrix element $(i, j)$ is set to 1 , if not, to 0 . As in the cluster method, 
TABLE 2: Computed Pauling Exponents and Configurational Entropies, $S,{ }^{25}$ for Extended Water Adlayers Periodic in One Dimension, as a Function of the Number of $\mathrm{O}$ Atoms in Both Sublattices of the Repeating Cell ${ }^{a}$

\begin{tabular}{ccc}
\hline O atoms in repeating cell & Pauling exponent & $S / k_{\mathrm{B}}$ \\
\hline 4 & 2.130735 & 0.756467 \\
6 & 2.123659 & 0.753141 \\
8 & 2.122934 & 0.752799 \\
10 & 2.122844 & 0.752757 \\
12 & 2.122832 & 0.752751
\end{tabular}

${ }^{a}$ Convergence is rapid, and the converged entropy is just $0.1 \%$ larger than the Pauling-like estimate, 0.752039.

there is always either no way or one way to place a molecule on a $\mathrm{B}$ site, once the orientations of the molecules on the surrounding three A sites are specified.

Given the way the $T$-matrix is constructed, the number of allowable arrangements of three chains (two A and one B between) is $\sum_{i, j} T(i, j)$, the number of allowable arrangements of five chains is $\sum_{i, j, k} T(i, j) T(j, k)$, and, in general, the number of allowable arrangements of $2 M+1$ chains is $\sum_{i, j} T^{M}(i, j)$. This last result makes it easy to obtain the number of allowed configurations in the limit that $M$ becomes large, since successive application of a matrix to an arbitrary vector refines out the eigenvector belonging to the matrix's largest eigenvalue. Accordingly, as $M \rightarrow \infty$, the entropy of the adlayer approaches $1 / 2 k_{\mathrm{B}} \ln \lambda_{\max }$, where $\lambda_{\max }$ is the largest eigenvalue of $T(i, j)$, and the factor $1 / 2$ represents the fact that the number of water molecules in the adlayer is twice the number on the A sublattice.

We present results obtained using Bartelt's transfer-matrix code in Table 2. Note that convergence is from above, and is rapid with respect to the repeat distance, $N / 2$, along the [112] chains. For $N=12$, the Pauling exponent is found to be $2.12283 \ldots$, close, as expected, to the Pauling-type estimate.

D. Vibrational Entropy in the Debye Model. The thermal energy of a $d$-dimensional solid whose phonons are purely acoustic is given by the Debye model expression ${ }^{26}$

$$
U(T)=d^{2} \Theta_{\mathrm{D}}\left(T / \Theta_{\mathrm{D}}\right)^{d+1} \int_{0}^{\Theta_{\mathrm{D}} / T} \frac{x^{d} \mathrm{~d} x}{\mathrm{e}^{x}-1}
$$

from which one obtains the constant-volume specific heat as $C_{V}=\mathrm{d} U / \mathrm{d} T$. The vibrational entropy is then

$$
S(T)=\int_{0}^{T} \frac{\mathrm{d} \tau}{\tau} C_{V}(\tau)
$$

Substituting eq D1 into D2 and interchanging the order of $\tau$ and $x$ integration allows the $\tau$ integral to be done explicitly, yielding the single-integral formula, eq 1 , in the main text.
Equation 1, incidentally, conveniently isolates the low- $T$ asymptotic contribution to the entropy in its second term. Thus

$$
\begin{aligned}
S(T \rightarrow 0 \mathrm{~K}) / N k_{\mathrm{B}}=d(d+1)\left(T / \Theta_{\mathrm{D}}\right)^{d} \int_{0}^{\infty} \frac{x^{d} \mathrm{~d} x}{\mathrm{e}^{x}-1} \equiv \\
d(d+1) !\left(T / \Theta_{\mathrm{D}}\right)^{d} \zeta(d+1)
\end{aligned}
$$

where $\zeta(d)$ is the Riemann zeta-function.

\section{References and Notes}

(1) Nagle, J. F. J. Math. Phys. 1966, 7, 1484.

(2) Lieb, E. H. Phys. Rev. 1967, 162, 162.

(3) (a) Hohenberg, P.; Kohn, W. Phys. Rev. 1964, 136, B864. (b) Kohn, W.; Sham, L. J. Phys. Rev. 1965, 140, A1133.

(4) Feibelman, P. J.; et al. J. Phys. Chem. B 2001, 105, 4018.

(5) See, e.g.: (a) Rydberg, H.; et al. Surf. Sci. 2003, 532, 606. (b) Wu, X.; et al. J. Chem. Phys. 2001, 115, 8748. (c) Lang, N. D. Phys. Rev. Lett. 1981, 46, 842.

(6) See, e.g.: Feibelman, P. J. Phys. Rev. B 2003, 67, 035420.

(7) Flubacher, P.; Leadbetter, A. J.; Morrison, J. A. J. Chem. Phys. 1960, 33, 1751. These authors determine that the entropy of ice increases by $0.308 \mathrm{~J} \mathrm{~K}^{-1} \mathrm{~mol}^{-1}$ between 0 and $15 \mathrm{~K}$. This is $9 \%$ of the zero-temperature residual entropy of ice Ih. Within a Debye model (see Appendix D), the vibrational entropy vanishes as $T^{d}$ as $T \rightarrow 0 \mathrm{~K}$, where $d$ is the dimensionality of the ice crystal, equal to 1,2 , or 3 .

(8) Pauling, L. J. Am. Chem. Soc. 1935, 57, 2680.

(9) Feibelman, P. J. Science 2002, 295, 99.

(10) Quoting Dorsey (Dorsey, N. E. Properties of Ordinary Water Substances; Reinhold: New York, 1940), Johari (Johari, G. P. J. Chem Phys. 2000, 112, 10957, Figure 2) says that, at $150 \mathrm{~K}$, the absolute entropy of bulk ice is $\sim 23.5 \mathrm{~J} \mathrm{~K}^{-1} \mathrm{~mol}^{-1}$ or about 7 times the residual entropy. Thus, at $150 \mathrm{~K}$ the residual entropy is $\sim 1 / 6$ the vibrational contribution.

(11) Held, G.; Menzel, D. Surf. Sci. 1994, 316, 92.

(12) Thiel, P. A.; Madey, T. E. Surf. Sci. Rep. 1987, 7, 211.

(13) Feibelman, P. J. Phys. Rev. B 2003, 67, 035420.

(14) Bernal, J. D.; Fowler, R. H. J. Chem. Phys. 1933, 1, 515.

(15) Note that the $l$ th layer attaches to the $(l-1)$ th by forming $M$ hydrogen bonds. Thus the number of missing bonds remains $M$, no matter how many layers are added-and is not $2 M$, despite the fact that a slab has two surfaces.

(16) If the first layer on the solid is part-dissociated, this argument may fail. The reason is that, till now, no one knows how water molecules would attach to a part-dissociated adlayer, and thus no one knows how to count the equal energy structures in this case.

(17) It is worth noting that, to a good approximation, the Debye entropies are independent of whether the system is constituted of $\mathrm{H}_{2} \mathrm{O}$ and $\mathrm{D}_{2} \mathrm{O}$ molecules. The reason is that the acoustic modes whose contribution the Debye model represents correspond to essentially rigid displacements of the water molecules. This is experimentally verified, in that phonon energy differences are minor, e.g., $\sim 5 \%$ lower for $\mathrm{D}_{2} \mathrm{O}$, reflecting its $11 \%$ higher mass. See: Graham, A. P.; Menzel, A.; Toennies, J. P. J. Chem. Phys. 1999, $111,1169$.

(18) Leadbetter, A. J. Proc. R. Soc. London, A 1965, A287, 403.

(19) Hobbs, P. V. Ice Physics; Clarendon Press: Oxford, 1974.

(20) For thick ice films, Braun (Braun, J.; et al. Phys. Rev. Lett. 1998, $80,2638)$ and Graham et al. ${ }^{17}$ obtain $\Theta_{\mathrm{D}}=175$ and $132 \mathrm{~K}$.

(21) For a single layer of $\mathrm{D}_{2} \mathrm{O} / \mathrm{Ru}(0001)$, a value of $\Theta_{\mathrm{D}}=193 \pm 50 \mathrm{~K}$ has been derived by G. Held (private communication) from data in Puisto et al. (Puisto, S. R.; Lorotholi, T. J.; Held, G.; Menzel, D. Surf. Rev. Lett. 2003, 10, 487).

(22) See: Onsager, L.; Dupuis, M. In Rendiconti della Scuola Internationale "Enrico Fermi", Corso X, Varenna; Zanichelli, N., Ed.; Bologna, 1959; p 294.

(23) $54=6^{3} / 2^{2}$. The Pauling denominator is $2^{2}$, because there are only two bonds in the linear case, not three.

(24) The Pauling denominator for the cyclic case is $2^{3}$, because there are three H-bonds.

(25) Bartelt, N. C. Unpublished.

(26) Dalvit, D. A. R.; Frastai, J.; Lawrie, I. D. Problems on Statistical Mechanics; IOP Publishing: Bristol, 1999. 